\title{
An Analysis of Factors Affecting the Duration of Latency Period and Its Impact on Neonatal Outcome in Patients with PPROM
}

\author{
Seema Singhal, Manju Puri, Neha Gami
}

\begin{abstract}
Introduction: Preterm premature rupture of membranes (PPROM) complicates approximately $3 \%$ of all births, but accounts for $30 \%$ of neonatal morbidity and mortality among premature gestations. Prediction of latency period for women with PPROM is imprecise and therefore consulting women with PPROM about their predicted latency period is a difficult task. The studies are limited, thus more information is required to support clinical decisions and to provide prognostic information in cases of expectant management following PPROM.
\end{abstract}

Materials and methods: We conducted a prospective observational study of women with singleton pregnancies presenting with rupture of membranes. A total of 120 women presenting with PPROM from 26 to 36 weeks with rupture of membrane were included in this study.

Results: Advanced maternal age $>30$ years was found to be associated with prolongation of latency period $(p=0.000)$. Nulliparity was found to be associated with shortening of latency period ( $p=0.012)$. An inverse association between gestational age at the time of presentation and latency period was established. The average gain in duration of latency period by not doing a digital examination was found to be statistically significant $(p=0.000)$. Gestational age and duration of latency period were found to be the important predictors of neonatal outcome.

Conclusion: In the current study, several predictive factors were identified which affect the duration of the latency period in cases of PPROM. This information may assist clinician in risk stratification and in providing consultation regarding the natural course of expectant management for women presenting with PPROM.

Keywords: Preterm premature rupture of membranes, Latency period, Neonatal outcome, Prematurity.

How to cite this article: Singhal S, Puri M, Gami N. An Analysis of Factors Affecting the Duration of Latency Period and Its Impact on Neonatal Outcome in Patients with PPROM. Int J Infertility Fetal Med 2012;3(3):87-91.

Source of support: Nil

Conflict of interest: None

\section{INTRODUCTION}

Preterm premature rupture of membranes (PPROM) complicates approximately $3 \%$ of all births, but accounts

Date of Received: 02-10-12

Date of Acceptance: 11-10-12

Date of Publication: September 2012 for $30 \%$ of neonatal morbidity and mortality among premature gestations. ${ }^{1,2}$ The main reasons are thought to be prematurity and infections. It has been seen that in women with PPROM 50\% will go into labor within 24 to 48 hours and 70 to $90 \%$ within 7 days. ${ }^{1}$ Latency period is defined as the interval between rupture of membranes and delivery. ${ }^{1}$ Currently the ability to predict the natural course and the duration of latency period is limited, and this makes counseling a difficult task. Expectant management of PPROM includes use of antibiotic treatment and steroids. ${ }^{1}$ Basic aim of expectant management is to prolong the pregnancy for possible longer durations because of the strong association between perinatal outcome and gestational age.

Aim of this study is to identify the factors associated with prolonged duration of latency period and to determine the neonatal outcome associated with longer latency periods in pregnancy with PPROM.

\section{MATERIALS AND METHODS}

We conducted a prospective observational study of women with singleton pregnancies who presented with rupture of membranes. The study was approved ethically. A total of 120 women presenting with PPROM from 26 to 36 weeks with rupture of membrane were included in this study. Rupture of membranes was confirmed by per speculum examination. Period of gestation was confirmed by accurate dating and/or first-trimester ultrasound. Only low-risk patients were included in the study. Patients who had cerclage operation, intrauterine death at the time of presentation, fetal anomalies, imminent delivery (defined as cervical examination $>6 \mathrm{~cm}$ on evaluation with regular uterine contractions), nonreassuring fetal or maternal status, clinical chorioamnionitis were excluded from the study. Maternal parameters like parity, maternal age at the time of admission, gestational age at the time of presentation were noted. Status of digital cervical examination whether not done or done before arrival to our center/in our center at the time of admission was recorded. After confirmation of PPROM, women were assessed for signs of labor, placental abruption and chorioamnionitis. If none of these were present they were put on expectant management. Latency period was defined as the time between admission due to rupture of membranes and time of delivery in days. In the 
course of hospital stay all women were managed by administration of antenatal steroids and antibiotics. The antimicrobial drug chosen was left to the discretion of the attending obstetrician. No tocolysis was performed. A four quadrant ultrasound for amniotic fluid index (AFI) and total leukocyte count was done after admission to the hospital. Antenatal monitoring was done by biophysical score and nonstress test. Latency for purpose of analysis was stratified into three groups $\leq 48$ hours, $>48$ hours to 1 week, $>1$ week. After delivery perinatal outcome was assessed. A note was made of birth weight, Apgar score at 1 and 5 minutes, duration of NICU stay and perinatal mortality. Neonatal morbidity outcome measures were recorded. Respiratory morbidity was defined as requirement of ancillary neonatal ventilator support or a positive chest $\mathrm{X}$-ray. Intraventricular hemorrhage was evaluated by neonatal cranial sonograms. Neonatal sepsis was considered, if blood cultures were positive within first 48 hours after birth. Data analysis was performed and one-way analysis of variance (ANOVA) was used to compare continuous variables between groups, and Chi-square test was used for categorical variables.

\section{RESULTS}

In present study a total of 120 patients were included. Several maternal characteristics were evaluated to determine, if they had any clinically significant impact on latency. The mean age of women presenting with PPROM was 27.3 years. The mean gestational age at which PPROM occurred was $31.96 \pm 2.90$ weeks. We observed that 87 out of 120 , i.e. $72.5 \%$ of the patients were $\leq 30$ years of age and $60 \%$ of women in this cohort were nulliparous. Advanced maternal age $>30$ years was found to be associated with prolongation of latency period $(p=0.000)$. Nulliparity was found to be associated with shortening of latency period $(\mathrm{p}=0.012)$.

We found that patients with PPROM, who were managed expectantly, 51.66\% delivered within 48 hours and $14.16 \%$ remained undelivered after a week from presentation. In patients who presented with rupture of membranes before 28 weeks of gestation, we observed that $31.25 \%$ delivered within 48 hours and $37.5 \%$ prolonged their latency by $>1$ week. However, the patients who presented at later gestations majority delivered earlier and only a small percentage prolonged their latency $>1$ week. The group which presented after 34 weeks, $80 \%$ delivered within 48 hours and none of them remained undelivered after 1 week. Therefore, we could establish an inverse association between gestational age at the time of presentation and latency period (Table 1 ), which was found to be statistically significant $(p=0.002)$. The mean latency period in our study group was $4.8439 \pm 6.55684$ days. The mean latency period for each group of gestational age also confirmed the same inverse association (Table 2) ( $p=0.000$, ANOVA).

The effect of digital cervical examination on latency period was also evaluated. We found that the patients in whom digital examination was documented before arriving to our center or was done in our center at the time of admission, latency period was short $<48$ hours in $76.36 \%$ of patients and $23 \%$ patients prolonged their latency by 48 hours. In this group only $1.81 \%$ women could prolong their latency by 1 week. On the other hand the group where digital cervical examination was not done $24.61 \%$ of women had latency period that was $>1$ week. This prolongation of latency period by not doing a digital cervical examination in patients who are kept on expectant management was found to be statistically significant $(p=0.000)$. The mean duration of latency period in the group where digital examination was done was $2.055 \pm 1.763$, and the group where digital examination was not done was $8.22 \pm 8.23$ days. When we evaluated the average gain in duration of latency period by not doing a digital examination, this gain was found to be statistically significant ( $p=0.000)$.

In our study, although it was seen that the patients who have decreased amniotic fluids, i.e. AFI $<5$ had prolonged latency period by more than 48 hours in $67.39 \%$ cases but the other group which had AFI $>5$ also showed prolongation of latency period in $63.15 \%$. This difference was not found to be statistically significant $(\mathrm{p}=0.45)$.

There are many factors which affect neonatal outcome in patients with PPROM. One such factor is gestational age at the time of PPROM. It is possible that in cases of PPROM, it is not only gestational age that should be taken into consideration, but also the duration of latency period through which the fetus is exposed to a potentially unfavorable intrauterine environment. Therefore, presence or absence of sepsis in neonate is a very important parameter of efficacy

\begin{tabular}{lccc} 
& Table 1: Effect of period of gestation (POG) on latency period \\
\hline Period of gestation & $\begin{array}{c}\text { Latency period } \leq 48 \text { hours } \\
(n=62)\end{array}$ & $\begin{array}{c}\text { Latency period }>48 \text { hours } \\
\text { to 1 week }(n=41)\end{array}$ & $\begin{array}{c}\text { Latency period }>1 \text { week } \\
(n=17)\end{array}$ \\
\hline $24-28$ weeks, $\mathrm{n}=16$ & $5(31.2 \%)$ & $5(31.2 \%)$ & $6(37.5 \%)$ \\
$28.1-32$ weeks, $\mathrm{n}=40$ & $16(40 \%)$ & $18(45 \%)$ & $6(15 \%)$ \\
$32.1-34$ weeks, $\mathrm{n}=34$ & $17(50 \%)$ & $12(34.6 \%)$ & $5(14.5 \%)$ \\
$34.1-36$ weeks, $\mathrm{n}=30$ & $24(80 \%)$ & $6(20 \%)$ & - \\
\hline
\end{tabular}


of expectant management. There was no statistically significant increase in neonatal sepsis (NNS) with prolongation of latency period ( $\mathrm{p}=0.4)$. We also noted a significant reduction in NNS with increase in gestational age $(p=0.01)$. Presence of neonatal morbidities also significantly reduced with increase in gestational age (Table 3) $(\mathrm{p}=0.05)$. However, prolongation of latency did not show any significant association with neonatal morbidities (Table 4). We could observe from the above study that the neonates who had presented with PPROM at earlier gestation had more chances of developing neonatal sepsis, which was as high as $75 \%$ in those who presented at $<28$ weeks gestational age at the time of rupture of membranes. However, on the other hand patients who presented at later gestations had significantly lesser chances of developing NNS. The group which presented at gestational age 32 to 34 weeks only $8.8 \%$ of neonates

\section{Table 2: Mean latency period according to POG}

\begin{tabular}{lc}
\hline Period of gestation & Mean latency period (days) \\
\hline 24-28 weeks, $\mathrm{n}=16$ & $9.88 \pm 9.05$ \\
28.1-32 weeks, $\mathrm{n}=40$ & $5.78 \pm 7.84$ \\
$32.1-34$ weeks, $\mathrm{n}=34$ & $4.13 \pm 4.435$ \\
$>34$ weeks, $\mathrm{n}=30$ & $1.69 \pm 6.55$ \\
\hline
\end{tabular}

showed signs of sepsis and the women who presented still later, i.e. between 34 to 36 weeks none of the neonates showed signs of sepsis.

Earlier gestational age at the time of PPROM is associated with earlier gestational age at the time of delivery. It has been demonstrated that, earlier the gestational age at the time of PPROM, worse is the neonatal outcome. As expected we found that, as gestational age increases, the risk of adverse neonatal outcomes in women receiving uniform administration of steroids and antibiotics decline, average duration of NICU stay decreased and the mean birth weight increased. However, at the same time prolonged latency did not influence the neonatal infectious morbidities significantly.

\section{DISCUSSION}

Prediction of latency period for women with PPROM is imprecise and therefore consulting women with PPROM about their predicted latency period is a difficult task. Our study found that the duration of latency period was inversely proportional to gestational age. In concordance with our findings previous studies have also noted a strong correlation between gestational age and latency period. ${ }^{1-4}$

\begin{tabular}{|c|c|c|c|c|}
\hline \multirow[t]{2}{*}{ Neonatal complications } & \multicolumn{4}{|c|}{ Period of gestation } \\
\hline & $\begin{array}{c}\leq 28 \text { weeks } \\
\quad(n=16)\end{array}$ & $\begin{array}{l}\text { 28.1-32 weeks } \\
\quad(n=40)\end{array}$ & $\begin{array}{l}\text { 32.1-34 weeks } \\
\quad(n=34)\end{array}$ & $\begin{array}{c}>34 \text { weeks } \\
(n=30)\end{array}$ \\
\hline $\operatorname{RDS}^{*}(n=15)$ & $10(66.66 \%)$ & $4(26.66 \%)$ & $1(6.66 \%)$ & - \\
\hline $\mathrm{IVH}^{\dagger}(\mathrm{n}=3)$ & $2(66.66 \%)$ & $1(33.33 \%)$ & - & - \\
\hline $\operatorname{ROP}^{\S}(n=2)$ & - & $2(100 \%)$ & - & - \\
\hline $\operatorname{NEC}^{\ddagger}(n=4)$ & $2(50 \%)$ & $1(25 \%)$ & $1(25 \%)$ & - \\
\hline Sepsis $(n=18)$ & $9(50 \%)$ & $6(33.33 \%)$ & $3(16.66 \%)$ & - \\
\hline Death $(n=12)$ & $10(83.33 \%)$ & $1(8.33 \%)$ & $1(8.33 \%)$ & - \\
\hline Mean NICUll & 11.45 days & 15.08 days & 7 days & 0.23 days \\
\hline$A / S^{* *}<7(n=17)$ & $9(52.94 \%)$ & $5(29.41 \%)$ & $3(17.64 \%)$ & - \\
\hline Birth weight (kg) & $0.946 \mathrm{~kg}$ & $1.51 \mathrm{~kg}$ & $1.829 \mathrm{~kg}$ & $2.32 \mathrm{~kg}$ \\
\hline Still birth $(n=4)$ & 4 & - & - & - \\
\hline
\end{tabular}

${ }^{*}$ Respiratory distress syndrome; ${ }^{\dagger}$ Intraventricular hemorrhage (only grade III or IV); ${ }^{\S}$ Retinopathy of prematurity; ${ }^{\ddagger}$ Necrotizing enterocolitis; "Neonatal intensive care unit stay; ${ }^{\star *}$ Apgar score

Table 4: Neonatal complications according to latency period

\begin{tabular}{|c|c|c|c|}
\hline Neonatal complications & $\begin{array}{l}\text { Latency period } \\
\leq 48 \text { hours }(n=62)\end{array}$ & $\begin{array}{l}>48 \text { hours }-1 \text { week } \\
(n=41)\end{array}$ & $\begin{array}{l}>1 \text { week } \\
(n=17)\end{array}$ \\
\hline $\operatorname{RDS}^{*}(n=15)$ & $7(46.66 \%)$ & $5(33.33 \%)$ & $3(20 \%)$ \\
\hline $\mathrm{IVH}^{\dagger}(\mathrm{n}=3)$ & $1(33.33 \%)$ & $1(33.33 \%)$ & $1(33.33 \%)$ \\
\hline $\operatorname{ROP}^{\S}(n=2)$ & $1(50 \%)$ & $1(50 \%)$ & - \\
\hline $\operatorname{NEC}^{\ddagger}(n=4)$ & $1(25 \%)$ & $2(50 \%)$ & $1(25 \%)$ \\
\hline Mortality (n = 12) & $5(41.66 \%)$ & $4(33.33 \%)$ & $3(25 \%)$ \\
\hline Mean NICU stay" (days) & 9.925 & 13.55 & 10.1875 \\
\hline $\mathrm{A} / \mathrm{S}^{\star *}<7(\mathrm{n}=17)$ & $7(41.17 \%)$ & $4(23.52 \%)$ & $6(17.64 \%)$ \\
\hline Mean birth weight $(\mathrm{kg})$ & 1.92 & 1.95 & 1.548 \\
\hline Still birth $(n=4)$ & 3 & - & 1 \\
\hline
\end{tabular}

\footnotetext{
${ }^{*}$ Respiratory distress syndrome; ${ }^{\dagger}$ Intraventricular hemorrhage (only grade III or IV); ${ }^{\S}$ Retinopathy of prematurity, ${ }^{\ddagger}$ Necrotizing enterocolitis;
}

"Neonatal intensive care unit stay; ${ }^{* *}$ Apgar score 
In present study advanced maternal age was found to be associated with prolongation of latency period. Nulliparity was found to be associated with shortening of latency. Similar association has also been noted in past. ${ }^{1,5}$

Advanced gestational age, digital cervical examination were found to be associated with significant shortening of latency. Gestational age at the time of rupture of membranes was inversely proportional to duration of latency. The duration of latency was significantly longer among women with PPROM before 34 weeks as compare to PPROM after 34 weeks. Mean latency period was found to be maximum in those women in whom rupture of membranes occurred before 28 weeks of gestation. Our finding was similar to other published studies ${ }^{1-4}$ but differed from Lieman et al who reported that the longest latency duration was between 28 to 31 weeks. ${ }^{6}$ The difference in findings could be because of difference in etiology of PPROM in two studied populations.

Digital cervical examination in a patient presenting with PPROM was found to be associated with significant reduction in duration of latency period $(\mathrm{p}=0.41)$. When compared with previous studies available in literature similar results were seen. ${ }^{7}$

The results of this study suggest that digital cervical examinations in patients with preterm PROM should be avoided until the clinician is convinced that the patient is clearly in labor. Digital cervical examinations shortens the duration of latency period, because it increases the incidence of chorioamnionitis and endometritis. ${ }^{7,8}$

Oligohydramnios has been associated with shortening of latency period. ${ }^{9}$ The reasons are many but most accepted one is that there is redistribution of blood flow in these fetuses because of fetal inflammatory response syndrome. This leads to oliguria and thus reduced production of liquor by the fetus, this inflammation also stimulates labor. Study by Gidon et al demonstrated that latency was prolonged in women who had oligohydramnios. ${ }^{10}$ The difference in observation was because of different study design in former and later group. However, our study could not show any association between oligohydramnios and duration of latency, this was probably because in almost $27.7 \%$ of patients in our study AFI was not documented at the time of admission. We suggest that AFI should be documented in patients with PPROM, as it is a very important predictor of duration of latency period.

The overall effect of latency period on neonatal outcome in women who present with PPROM can be considered as a fine balance between beneficial effects (e.g. more advanced lung maturity) and negative effects which may have resulted from prolonged exposure of fetus to an unfavorable intrauterine environment. Therefore, in order to determine a gestational age at which negative effects of expectant management outweigh its beneficial effects, we compared the outcome of cases of PPROM in relation to gestational age and latency period. At the same time we also tried to find out a gestational age at which pregnancy can be terminated safely with maximum neonatal benefits and no or minimal negative effects of prolonged latency period. The incidence of complications were more in fetuses who delivered within first 48 hours because probably these fetuses did not receive the benefit of expectant management and cumulated the worst conditions, such as young gestational age at birth, partial exposure to antibiotic and partial exposure to steroid treatment. This difference was not found to be statistically significant. In contrast the fetuses delivered after at least 48 hours should have received the entire effect of steroids, antibiotics and were older at birth. Our results suggested that for women who presented with PPROM the overall effect of expectant management is beneficial. Outcome of neonates who delivered after 34 weeks of gestation was optimum. There was no morbidity because of prematurity and infection and a significant increase was seen in birth weight with a significant reduction in duration of NICU stay. Thus, we suggest that immediate delivery after PPROM should not be practiced especially in developing countries like India, rather patients should be kept on expectant management for longer possible durations possibly 34 weeks or even up to 36 weeks. Our results were consistent with other studies in literature. ${ }^{11,12}$ Considering the effect on birth weight and NICU stay, prolongation of pregnancy even up to 36 weeks can be practiced at centers where optimum neonatal facilities are not readily available or where cost is the limiting factor. There were total of 12 neonatal mortalities in our study. Out of which 10 were of $<28$ weeks gestation. All these deaths occurred before discharge from initial hospitalization. In this group mean duration of latency was 17 days and the mean birth weight was 898.80 gm. Respiratory morbidity and sepsis screen was positive in all these neonates. Therefore, we could strongly say that neonatal morbidity and mortality in patients on conservative management after PPROM depends upon prematurity and low birth weight.

There are several limitations to our study. Although all patients were managed by a uniform policy of expectant management with standardized protocols for steroid and antibiotic administration, each was cared by a different caregiver. Timing of delivery in patients with PPROM in our institution is after 34 weeks gestation, and the ultimate decision to proceed with delivery is often dependent on the attending physician's decisions, this could have biased our results. Another important confounder could be presence of infection at the time of PPROM, which is a very important 
predictor of latency period but unfortunately we did not have information. Such infections could be screened by assessing amniotic fluid inflammatory markers. Major potential determinants of neonatal outcome birth weight, neonatal morbidities, failed to reveal a statistically significant impact on infant morbidity and mortality. This may be due to a relatively small number of cases in both groups. This study needs to be done at a larger scale.

\section{CONCLUSION}

In the current study, we have identified several predictive factors which affect the duration of the latency period in cases of PPROM. This information may assist clinician in risk stratification and in providing consultation regarding the natural course of expectant management for women presenting with PPROM. Despite limitations our findings regarding the inverse relationship of gestational age with latency duration, neonatal morbidity and mortality, and data presented can be useful for counseling the patients with PPROM about expected duration of latency and outcomes.

\section{REFERENCES}

1. Gopalani S, Krohn M, Meyn L, Hitti J, Crombleholme WR. Contemporary management of preterm premature rupture of membranes: Determinants of latency and neonatal outcome. Am J Perinatol 2004 May;21(4):183-90.

2. Manuck TA, Maclean CC, Silver RM, Varner MW. Preterm premature rupture of membranes: Does the duration of latency influence perinatal outcomes? Am J Obstet Gynecol 2009; 201(4):414

3. Aziz N, Cheng YW, Caughey AB. Factors and outcomes associated with longer latency in preterm premature rupture of membranes. J Matern Fetal Neonatal Med 2008;21(11): 821-25.

4. Melamed N, Hadar E, Ben-Haroush A, Kaplan B, Yogev Y. Factors affecting the duration of the latency period in preterm premature rupture of membranes. J Matern Fetal Neonatal Med 2009;22(11):1051-56.

5. Tanir HM, Sener T, Tekin N, Aksit A, Ardic N. Preterm premature rupture of membranes and neonatal prior to 34 weeks of gestation. Int J Gynaecol Obstet 2003;82(2):167-72.
6. Lieman JM, Brumfield CG, Carlo W, Ramsay PS. Preterm premature rupture of membranes: Is there an optimal gestational age for delivery? Obstet Gynecol 2005;105:12-17.

7. Lewis DF, Major CA, Towers CV, Asrat T, Harding JA, Garite T. Effects of digital vaginal examination on latency period in preterm premature rupture of membranes. Am J Obstet Gynecol 1992;80:630-34.

8. Royal College of Obstetricians and Gynaecologists. Preterm premature rupture of membranes. London (UK): Royal College of Obstetricians and Gynaecologists (RCOG); 2006 Nov:11.C (Guideline no. 44)

9. Park JS, Yoon BH, Romero R, Moon JB, Oh SY, Kim JC, Jun $\mathrm{JK}$. The relationship between outcome oligohydramnios and the onset of preterm labor in preterm premature rupture of membranes. Am J Obstet Gynecol 2001;184(3):459-62.

10. Test G, Levy A, Wiznitzer A, Mazor M, Holcberg G, Zlotnik A, et al. Factors affecting the latency period in patients with preterm premature rupture of membranes. Arch Gynecol Obstet 2011;283(4):707-10.

11. Pasquier JC, et al. Neonatal outcome after elective delivery management of preterm premature rupture of membranes before 34 weeks gestation (DOMINOS study). Eur J Obstet gynecol Reprod Biol 2009;143:18-23.

12. Guangyong YE, Jiang Z, Shiming LU, Yuanluo LE. Premature infants born after preterm premature rupture of membranes with 24-34 weeks of gestation : A study of factors influencing length of neonatal intensive care unit stay. J Maternal- Fetal Neonatal Med 2011 July;24(7):960-65.

\section{ABOUT THE AUTHORS}

\section{Seema Singhal}

Associate Professor, Department of Obstetrics and Gynecology, Lady Hardinge Medical College and SSK Hospital, New Delhi, India

Correspondence Address: F-60, Ansari Nagar (West), AIIMS Campus AIIMS, New Delhi, India, e-mail: drseemasinghal@gmail.com

\section{Manju Puri}

Professor, Department of Obstetrics and Gynecology, Lady Hardinge Medical College and SSK Hospital, New Delhi, India

\section{Neha Gami}

Assistant Professor, Department of Obstetrics and Gynecology, Lady Hardinge Medical College and SSK Hospital, New Delhi, India 